\title{
Rotor Faults Diagnosis Using Artificial Neural Networks and Support Vector Machines
}

\author{
Sukhjeet Singh and Navin Kumar \\ School of Mechanical, Materials and Energy Engineering, Indian Institute of Technology Ropar, Nangal Road, \\ Rupnagar, Punjab, India
}

\begin{abstract}
(Received 3 June 2013; accepted 1 January 2014)
Unbalance and misalignment are the commonly occurring faults in rotating mechanical systems. These faults are caused mainly due to improper installation or premature failure of the machine components. Detection and diagnosis of faults in rotating machinery is crucial for its optimal performance. In this study artificial neural networks (ANN) and support vector machine (SVM) techniques have been used to determine the effectiveness of statistical features for fault diagnosis in rotating mechanical system using healthy and faulty rotors. The vibration signature responses are obtained and analyzed for healthy shaft without disk (HSWD), healthy shaft with an unbalanced disk (HSWUD), centrally bent shaft without disk (CBSWD) and centrally bent shaft with an unbalanced disk (CBSWUD) with zero bow phase angle. Their predominant features were fed as input for training and testing ANN and SVM, whereas the relative efficiency of these techniques have been compared for classifying the faults in the test system. The study concludes that these machine learning algorithms can be used for fast and reliable diagnosis of rotor faults.
\end{abstract}

\section{NOMENCLATURE}

\begin{tabular}{|c|c|}
\hline$T$ & Bias or threshold \\
\hline$\lambda_{i}$ & Lagrange multipliers \\
\hline$U(\lambda)$ & Lagrange function \\
\hline$\phi_{j}^{h}$ & Bias for hidden layer \\
\hline$n e t_{m n}^{h}$ & Net input to hidden layer \\
\hline$n e t_{m k}^{o}$ & net input to output layer \\
\hline$\mu_{m n}$ & $n^{\text {th }}$ input of the $m^{\text {th }}$ input vector \\
\hline$\kappa_{i}$ & $\begin{array}{l}\text { Distance between the margin and the examples } \\
\mu_{i} \text { that are lying on the wrong side } \\
\text { of the margin }\end{array}$ \\
\hline$\phi_{j}^{o}$ & Bias for output layer \\
\hline$E_{m}$ & Sum of squares error \\
\hline$O_{m k}^{o}$ & Output of output layer \\
\hline$V_{m}$ & Sum of squares error \\
\hline$\triangle_{m}^{o}$ & Change in weight w.r.t weight change \\
\hline$O_{m j}^{h}$ & Output of hidden layer \\
\hline$u_{j k}^{h}$ & $\begin{array}{l}\text { Synaptic weight between hidden } \\
\text { and output layer }\end{array}$ \\
\hline M & Number of iterative step \\
\hline$Z_{m k}$ & Desired output \\
\hline
\end{tabular}

\section{INTRODUCTION}

Rotating machinery diagnostics is an essential function in industrial processes and power generation applications. Failures in a rotating machinery system are quite common and their proper diagnosis depends upon accurate detection of the fault and its location. Most of the faults are caused either because of the incorrect manufacturing practices or because of the extreme operating conditions. These may result in excessive heat generation, looseness and other unwanted wears and tears of the rotating parts causing financial losses. Therefore, a contin- uous monitoring system is required to detect and diagnose the faults to avoid any such situation.

Faults associated with the rotor-bearing system like unbalanced rotor, $; 1,2$ bent rotor, $; 3$ misaligned rotor, ${ }^{4}$ and rotor rub ${ }^{5,6}$ are discussed in the literature. Many techniques and tools are already in practice for the continuous diagnosis of the various components of the rotating machinery. $\mathrm{Li}$ et al. ${ }^{7}$ used the hidden Markov models (HMM) techniques in order to detect various faults namely: rotor unbalance, rotor to stator rub, oil whirl and pedestal looseness in a rotating machinery under speed-up and speed-down conditions. Rolling element bearings defects like outer race, inner race, ball spin and cage faults were discussed by different researchers ${ }^{8-12}$ using different techniques. Also, back-propagation learning algorithm and a multi-layer network have been used to validate the test data for unknown faults. $^{13}$

Fault diagnosis of load machines like gearboxes for common defects like missing tooth and wear of the gear tooth were carried out using wavelet techniques. ${ }^{14}$ KolmogorovSmirnov test was used by Kar and Mohanty for the detection of faulty gears. ${ }^{15}$ The performance of gear fault was detected using ANN and SVM by Samanta. ${ }^{16}$ Support vector machines (SVM) were used in the fault diagnosis of machines. ${ }^{17}$

The present work deals with the extraction of statistical features from the vibration signatures of a rotor-bearing system and classification of shaft faults using artificial neural network (ANN) and support vector machine (SVM). The flow chart for the shaft health diagnosis is shown in Fig. 1.

A group of statistical features like range, root mean square value, crest factor, kurtosis, skewness and standard deviation have been extracted from time domain. The setup details for simulating the combined unbalance and bent rotor fault in a real experimental machine have been discussed in Section 2. 